\title{
Rotated infrared antenna transmitarray for the manipulation of circularly polarized wavefronts
}

\author{
Yuchu $\mathrm{He}^{*}$ and George V. Eleftheriades \\ Department of Electrical and Computer Engineering, University of Toronto, Canada
}

Received 28 March 2014 / Accepted 16 February 2015

\begin{abstract}
An IR optical transmitarray is presented based on Antenna Array Sheet (AAS) for the manipulation of circularly polarized light. The unit cell of the transmitarray comprises three layers of metallic elliptical patches. Complete phase control is achieved through the local rotation of each unit cell. Thin refraction and focusing transmitarrays of this sort are demonstrated at infrared frequencies. Moreover, a new concept for realizing a polarization-discriminating device is introduced based on a flat refracting lens. These devices are compatible with current fabrication technology and can become crucial for the integration with other IR and nano-photonic devices.
\end{abstract}

Key words: Metasurfaces, Transmitarrays, Infrared lens, Circular polarization, Polarization discrimination.

\section{Introduction}

The conventional method of optical wavefront control and manipulation often relies on bulky dielectric lenses. For example, a glass lens that is used to focus light has a polished parabolic surface such that light propagating through it experiences spatially-dependent delay and arrives at the focal point in phase. However, even with current nano-fabrication technology, it is still difficult to create a dielectric lens with parabolic or nonplanar surface at the $\mu \mathrm{m}$ scale to integrate with nano-photonic systems. To overcome this challenge, novel techniques have been proposed in [1-7]. In Yu et al. [1] and Aieta et al. [2], a two dimensional patterned structure was demonstrated to control the wavefront of light by using 2D arrays of V-shaped gold nano-antennas deposited on a silicon wafer. As the linearly polarized light impinges on the V-shaped antennas, symmetric and anti-symmetric currents are induced on the antennas such that the phase of the scattered light in the cross-polarization can be controlled while maintaining a constant scattering magnitude. Hence, the emerging wavefronts of the scattered light from the silicon interface are tailored into the desired pattern. The advantage of this concept is that it leads to a thin planar structure that can be easily realized with current fabrication technology. The disadvantage is though that the scattered light from those nano-antennas has a small intensity to be useful for practical applications. In $\mathrm{Ni}$ et al. [3], Babinet-inverted $\mathrm{V}$-shaped slot antennas are introduced for focusing the transmitted light in the cross-polarization. The working principal is similar to these in $[1,2]$, but the efficiency is improved. However, according to Monticone et al. [4], the fundamental upper limit

*e-mail: yhe@ece.utoronto.ca of the power that can be coupled into the cross-polarization of the scattered light is $50 \%$. Even if this cross-polarization coupling can be maximized, due to the symmetry of the design in [1-3], half the power is scattered into the forward direction and half into the backward direction. This implies that at most only $25 \%$ of the incident power can be coupled into the transmitted cross-polarization. To improve the transmission efficiency, Monticone et al. [4] introduced the concept of the meta-transmit-array which is similar to transmitarrays in the microwave regime. Three cascaded metasurfaces are used to provide full control of the transmission phase. The metasurface is composed of periodically arranged unit cells. Each unit cell consists of a block of a nonmetallic plasmonic material (aluminum-doped zinc oxide) and a block of a dielectric material (silicon). Tuning of the phase delay of each unit cell is achieved through changing the thicknesses of the two material blocks. The complexity and required accuracy of the design still remains a challenge for its realization with existing technologies. In Pfeiffer and Grbic [5], a more realizable lens was proposed. Each unit cell in this lens comprises four cascaded patterned metallic sheets with dimensions parameterized to obtain the required transmission phase and magnitude for two orthogonal polarizations. The structure can convert a linearly polarized incident light into circularly polarized one and achieve focusing at the same time. The drawback is that to keep the lens functional, the linearly polarized incident light has to be oriented at $45^{\circ}$ relative to the horizontal $X$-axis so that the $X$ and $Y$ components of the metasurface are equally excited. Moreover, the unit cell design has to be parameterized to achieve the required phase delays. In fact, the designs in [1-7] all use aperiodic unit-cell structures to tailor the phase component of the linearly polarized light. If a different phase 
requirement is imposed, the unit cells have to be re-designed and optimized. Such a design process can be very cumbersome and inflexible. In Chen et al. [8], a rotation technique has been introduced to simplify the phase tailoring process which is related to the concept presented earlier in Huang and Pogorzelski [9]. The unit cell in Chen et al. [8] consists of a plasmonic dipole made of gold. By rotating each dipole, the incident circularly polarized (CP) light is scattered into a right-hand circularly polarized (RHCP) and a left-hand circularly polarized (LHCP) light, with one of them having a controllable phase discontinuity associated with the dipole rotation. By using a quarter-wave plate (QWP) and a polarizer to filter out the controllable polarization, focusing can be achieved. This concept leads to a simpler phase control technique compared to the ones presented in [1-7]. However, there are two major drawbacks. First, as any single layer lens, the scattering efficiency has an upper bound as mentioned before. In this particular case, the efficiency is only $5 \%$, which deems the lens impractical for real world applications. Second, the transmitted light has both RHCP and LHCP light and only one of the polarization is controllable, the other one is not. Hence, with the uncontrollable polarization present, a QWP and a polarizer are needed for filtering, which ultimately undermines the potential for integration. Furthermore, the additional power loss introduced by the QWP and the polarizer is undesirable in imaging applications.

In this paper, we propose an optical transmitarray that tailors the transmission phase of the incident $\mathrm{CP}$ light by using the rotation technique similar to the one in Chen et al. [8] but this time we are able to greatly improve the transmission efficiency and eliminate the uncontrollable polarization. This makes our design very practical and amenable for integration since the QWP and the polarizer are not needed. The method of eliminating the uncontrollable polarization originates from a reflectarray design [9] for circular polarization in the microwave regime. Transmitarrays working in the microwave regime have been built in Phillion and Okoniewski [10] based on the method proposed in Huang and Pogorzelski [9]. However, it is difficult to directly translate these designs to the optical regime. The unit cell in Phillion and Okoniewski [10] consists of five layers of metallic structures. The top and bottom two layers are rectangular patches. The middle layer is a metallic sheet with orthogonal slots, which are used to couple the top and bottom patches. This makes it difficult to directly translate this design into the optical regime. The achievable fabrication resolution might not reach the required accuracy for the aperture size and this will have a great negative impact on the performance of the transmitarray. In this work, we can achieve the required phase delay by using only three metallic layers instead of five, without using the aperture coupling mechanism of Phillion and Okoniewski [10]. Therefore, the fabrication complexity of our structure is simpler and can be readily realized in the optical regime.

The unit cell that we use is based on our recently introduced thin wave-plates comprising three layers of antenna array sheets AAS [11]. These AAS consist of elliptical metallic patches in a dielectric matrix. By rotating each triple-patch unit cell, the transmitted wave of each unit cell is delayed by a phase that is directly proportional to its local rotation angle. This is very appealing because one simply has to optimize the design for one unit cell and achieve arbitrary phase delay

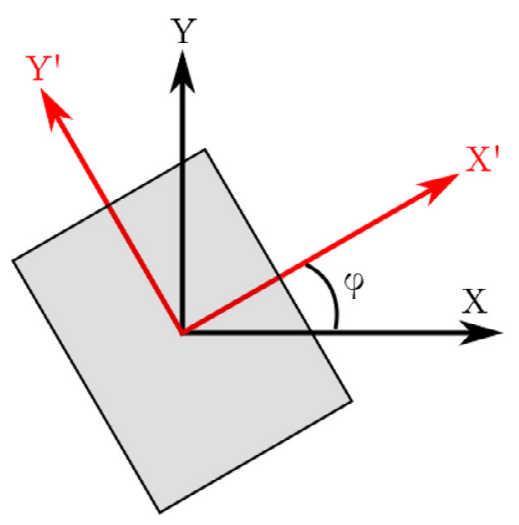

Figure 1. Rotated unit cell with respect to the original (unprimed) and the new (primed) coordinate system. The rotation angle is $\varphi$.

just by rotation. This offers simplicity and flexibility to the design process. In this paper, this concept is demonstrated by designing optical transmitarrays for refraction and focusing working at the long wavelength infrared (LWIR) region with a design frequency of $30 \mathrm{THz}$. High transmission and low reflection can be achieved over a reasonable bandwidth of $14 \%$. The proposed design is compatible with existing fabrication technology so that it can be easily integrated. The lens offers control for circularly polarized light which is very appealing for industrial $\mathrm{CO}_{2}$-laser machining at $10.6 \mu \mathrm{m}$ because circularly polarized light offers more uniform and efficient cutting [12]. The materials used in this design have high laser-induced damage threshold (LIDT) which is especially desired for high-power laser machining applications. It should be noted that there is no constraint on the orientation of the linearly polarized incident beam as in the prior art. As long as the incident beam is circularly polarized, the lens remains functional. Lastly, we are introducing a new polarization discriminator concept: a flat refraction lens can be used to detect the orientation angle of a linearly polarized plane wave at normal incidence by simply measuring the spatial variation of the transmitted intensity. Such concept can be very attractive for potential infrared polarimetric imaging applications [13].

\section{Theory and design}

\subsection{Theory}

Suppose the incident field is LHCP propagating in the $+z$ direction, in an unprimed $X-Y$ coordinate system, the incident field is given by equation (1) in time harmonic form with $e^{j \omega t}$ terms omitted.

$$
\vec{E}_{\mathrm{inc}}=\hat{x} E_{x}+j \hat{y} E_{y}
$$

Once the incident field impinges on the unit cell, it may experience changes in both the amplitude and phase in the $X$ and $Y$ polarizations, then the transmitted field $\vec{E}_{t}$ is given by equation (2):

$$
\vec{E}_{t}=\hat{x} E_{x}^{\prime} e^{j \theta_{x}}+j \hat{y} E_{y}^{\prime} e^{j \theta_{y}}
$$

Let the unit cell (gray patch) as shown in Figure 1 be rotated by an angle $\varphi$ with respect to the original (unprimed) 


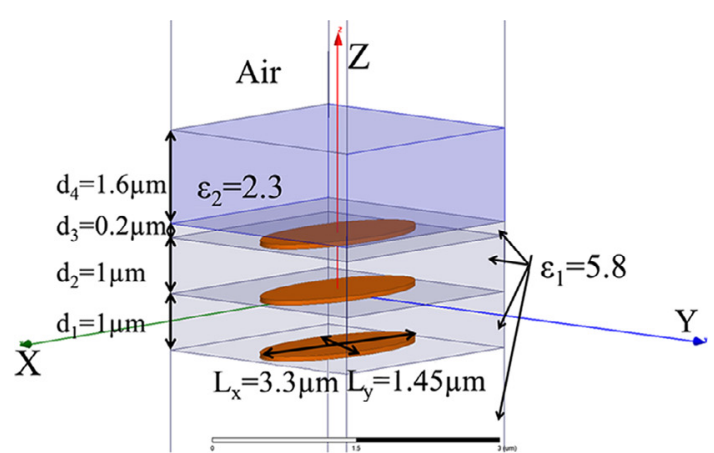

(a)

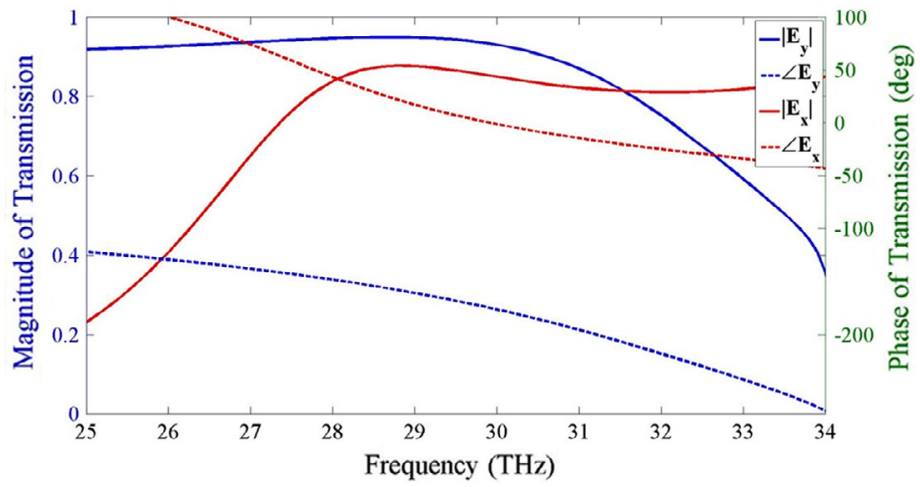

(b)

Figure 2. (a) The unit cell design of the half-wave plate that comprises three metallic elliptical patches and interlayer dielectrics, (b) transmission of the half-wave plate design for the $X$ and $Y$ polarizations.

coordinate system and aligned to the new (primed) coordinate system. The incident field can be written in the new coordinate system as in equation (3):

$$
\begin{aligned}
\vec{E}_{\text {inc }}= & \left(\hat{x}^{\prime} E_{x} \cos \varphi-\hat{y}^{\prime} E_{y} \sin \varphi\right) \\
& +j\left(\hat{x}^{\prime} E_{x} \sin \varphi+\hat{y}^{\prime} E_{y} \cos \varphi\right) \\
= & \hat{x}^{\prime} E_{x} e^{j \varphi}+j \hat{y}^{\prime} E_{y} e^{j \varphi}
\end{aligned}
$$

Then the transmitted field simply becomes:

$$
\vec{E}_{t}=\hat{x}^{\prime} E_{x}^{\prime} e^{j \theta_{x}} e^{j \varphi}+j \hat{y}^{\prime} E_{y}^{\prime} e^{j \theta_{y}} e^{j \varphi}
$$

We can further rewrite $\hat{x}^{\prime}$ and $\hat{y}^{\prime}$ in terms of $\hat{x}$ and $\hat{y}$, then $\vec{E}_{t}$ can be written as:

$$
\begin{aligned}
\vec{E}_{t}= & (\hat{x} \cos \varphi+\hat{y} \sin \varphi) E_{x}^{\prime} e^{j \theta_{x}} e^{j \varphi} \\
& +j(-\hat{x} \sin \varphi+\hat{y} \cos \varphi) E_{y}^{\prime} e^{j \theta_{y}} e^{j \varphi}
\end{aligned}
$$

After some algebra, we have:

$$
\begin{aligned}
\vec{E}_{t}= & \frac{1}{2}\left(E_{x}^{\prime} e^{j \theta_{x}}-E_{y}^{\prime} e^{j \theta_{y}}\right) e^{j 2 \varphi}(\hat{x}-j \hat{y}) \\
& +\frac{1}{2}\left(E_{x}^{\prime} e^{j \theta_{x}}+E_{y}^{\prime} e^{j \theta_{y}}\right) \hat{x}+j \hat{y}
\end{aligned}
$$

In equation (6), we have written the transmitted wave as a summation of LHCP and RHCP waves whereas the incident wave is LHCP. Only the first term, i.e. the RHCP wave has a phase associated with the element rotation $\varphi$. In order to control the phase of the transmitted wave by directly rotating the element, the second term in equation (6) has to be eliminated. The required conditions are:

$$
\left\{\begin{array}{r}
E_{x}^{\prime}=E_{y}^{\prime} \\
\theta_{x}=\theta_{y}+\pi
\end{array}\right.
$$

If the above amplitude and phase constraints are satisfied, the transmitted field can be written as in equation (8). It is clear that the phase of $\vec{E}_{t}$ is directly controlled by the rotation angle

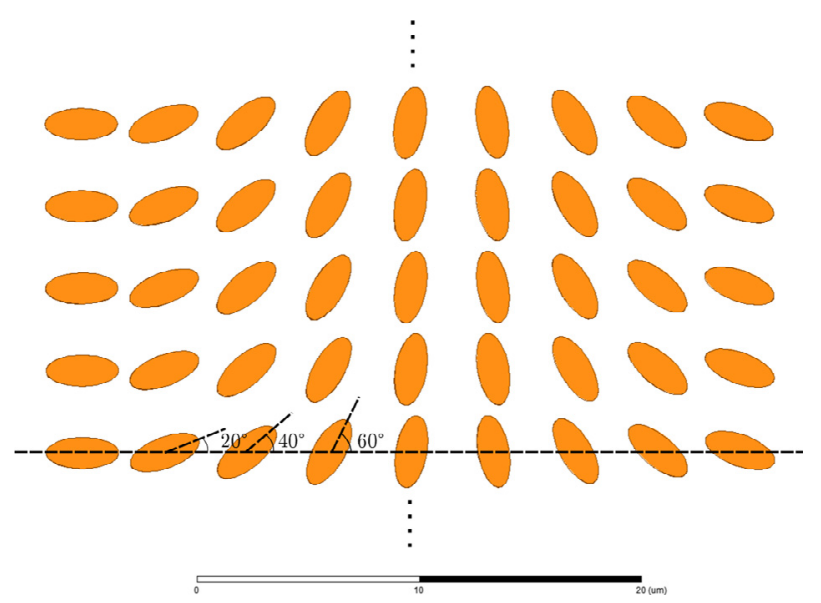

Figure 3. Top view of the refraction transmitarray. Unit cells from left to right have an incremental rotation angle of $20^{\circ}$.

$\varphi$, however, the handedness of the polarization is converted. If the incident wave is LHCP, then the transmitted wave would be RHCP and vice versa.

$$
\vec{E}_{t}=E_{x}^{\prime} e^{j \theta_{x}} e^{j 2 \varphi}(\hat{x}-j \hat{y})
$$

\subsection{Element design}

To satisfy the constraints in equation (7), the unit cell should behave like a half-wave plate. Such a design can be implemented by using cascaded antenna array sheets [11] as shown in Figure 2a. This unit cell comprises elliptical patches made of $50 \mathrm{~nm}$ thick gold and dielectrics materials of $\mathrm{ZnSe}$ and $\mathrm{YbF}_{3}$. Such materials have high LIDT [14] for high power laser applications. The design can be fabricated by focused ion beam (FIB) and chemical vapor deposition (CVD). The transmission of such design is shown in Figure 2b. At the operating frequency, the transmission phase difference between the $X$ and $Y$ polarized field is $180^{\circ}$ and the transmission magnitudes are about 0.9 . The transmission efficiency of this unit cell is $80 \%$ at operating frequency. 


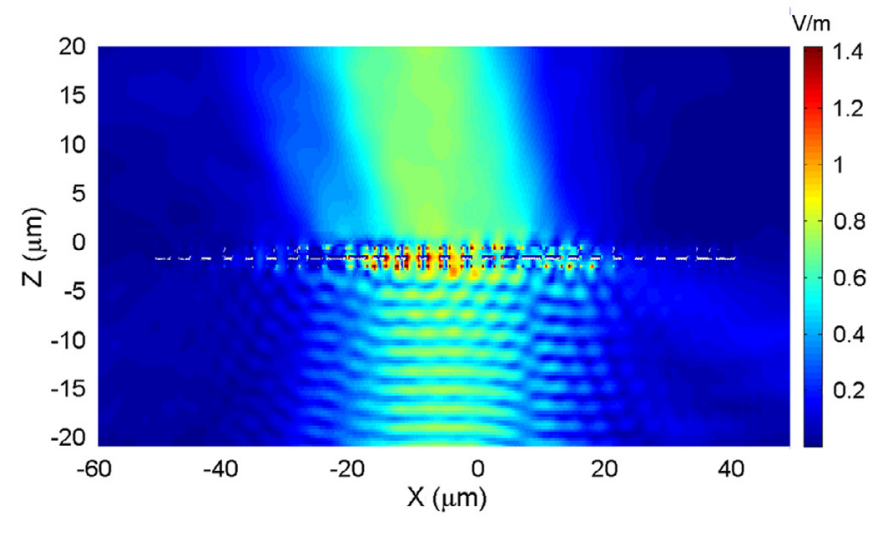

(a)

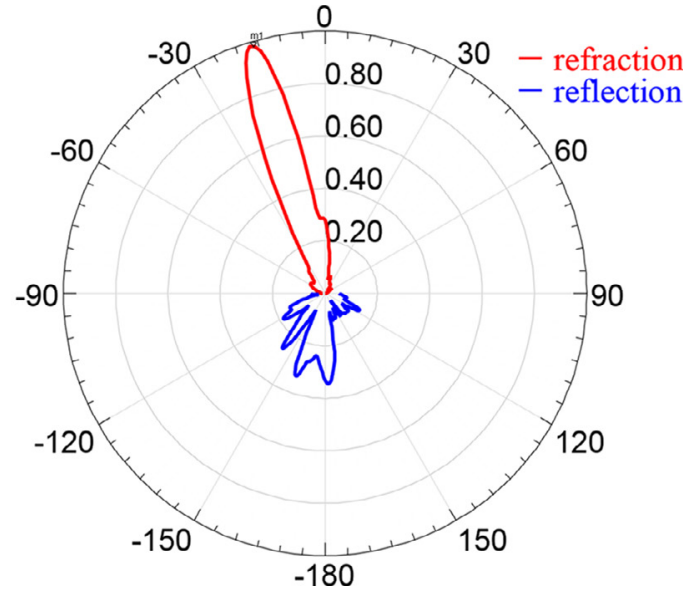

(b)

Figure 4. Transmitted RHCP wave from a LHCP Gaussian beam at normal incidence. (a) Peak magnitude of the incident beam is $\sqrt{2} \mathrm{~V} / \mathrm{m}$, (b) normalized far-field pattern of the refracted and reflected beams.

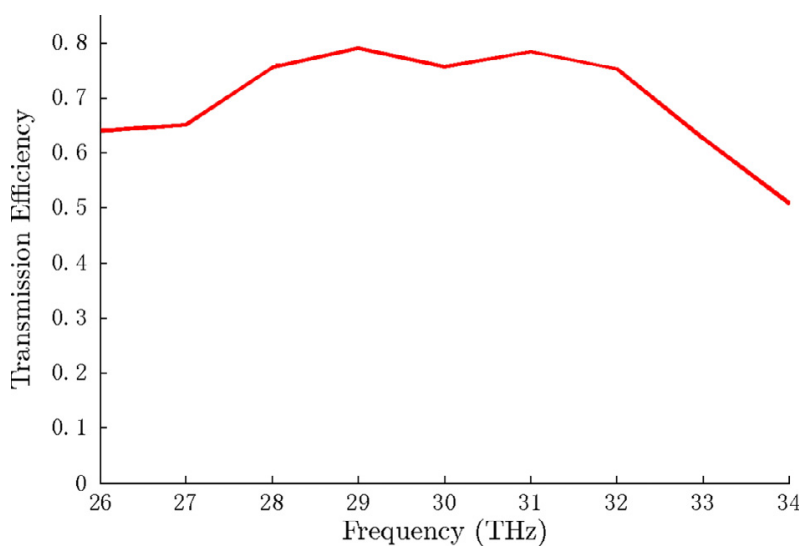

Figure 5. Over $70 \%$ transmission efficiency can be maintained over the frequency range of $28-32 \mathrm{THz}$. This is equivalent to $14 \%$ bandwidth.

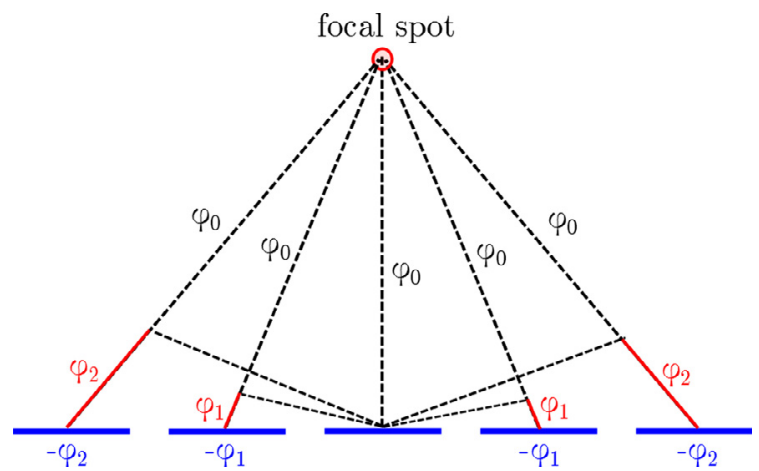

Figure 6. The central element has a free space path delay of $\varphi_{0}$. The adjacent elements have additional free space path delays of $\varphi_{1}$ and $\varphi_{2}$. By rotating the corresponding unit cells with phase delays of $-\varphi_{1}$ and $-\varphi_{2}$, the path delays can be compensated and the transmitted waves from each unit cell will arrive at the focal spot in phase.

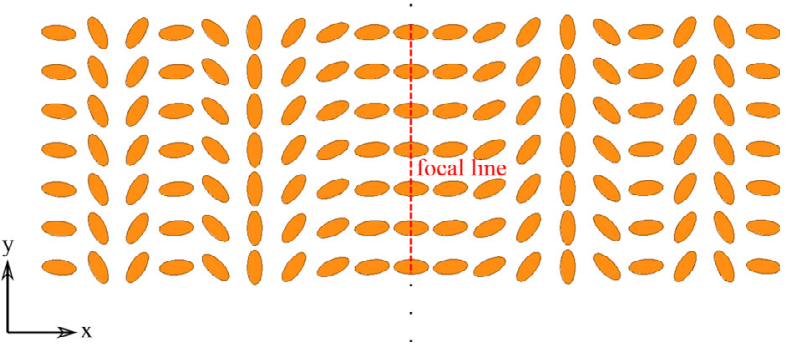

Figure 7. The 1D-focusing transmitarray is designed to focus the incident normal beam into a focal line at the center (red dashed line). Each unit cell is rotated according to its position to compensate the free space path delay.

\subsection{Applications: 1D-refraction}

From the Huygens principle, the wavefront can be reconstructed from the spherical wavelets of the constituent Huygens sources. Refraction arises if there is a constant phase delay between successive Huygens sources. By treating each unit cell as a Huygens source and manipulating the phase delay of each individual unit cell, we are able to create a transmitarray that can refract a normally incident wave to an arbitrary angle. With the unit cell design presented in Section 2.2, the required phase delay can be achieved by rotating each unit cell. Figure 3 shows the top view of such a transmitarray. As shown, the unit cells from left to right have an incremental rotation angle of $20^{\circ}$. Hence, each unit cell can introduce an additional $40^{\circ}$ phase shift compared to the adjacent unit cell on the left. The simulated result of this transmitarray is shown in Figures $4 \mathrm{a}$ and 4b. The transmitted beam corresponds to a refraction angle of $17^{\circ}$. Figure 5 shows the extracted transmission efficiency vs. frequency of the transmitarray. Above $70 \%$ efficiency can be maintained over a $14 \%$ bandwidth (28-32 THz). At the operating frequency, the transmission efficiency is $76 \%$. It should be noted that the efficiency of the entire transmitarray agrees well with 


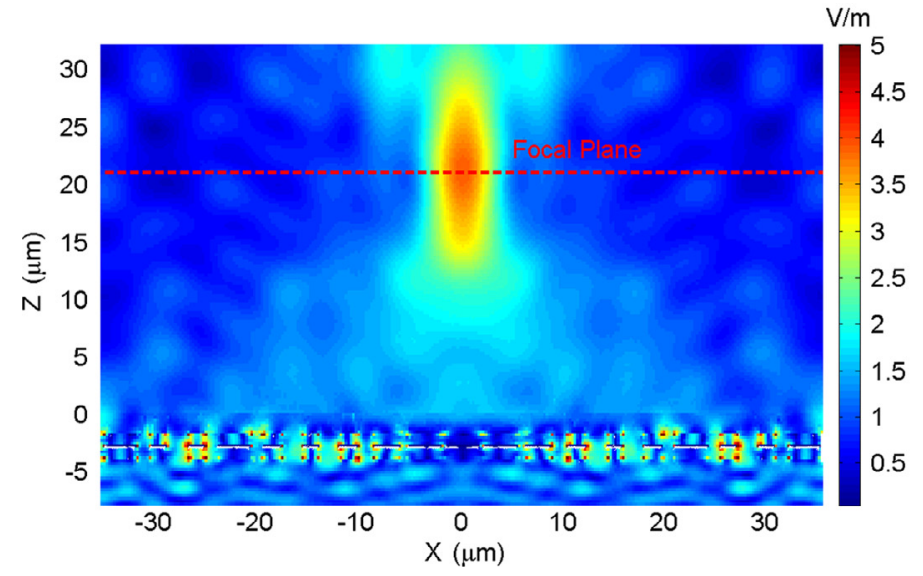

(a)

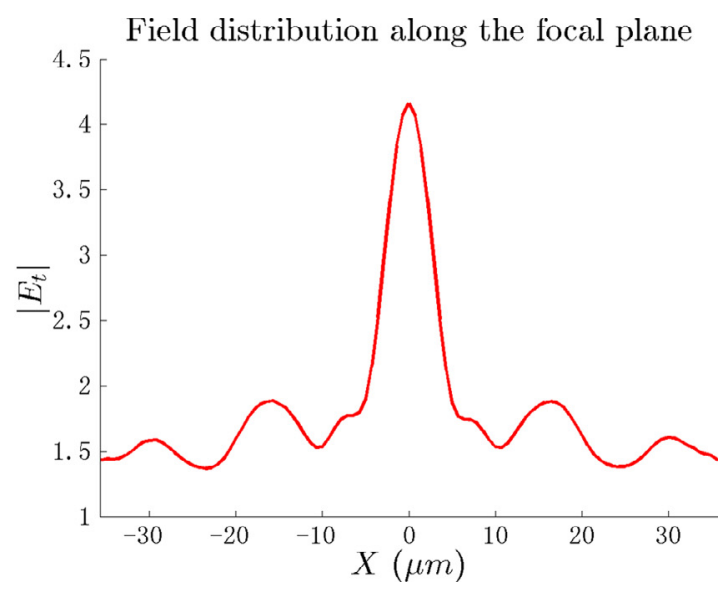

(b)

Figure 8. Complex magnitude of the transmitted field. (a) The simulated focal length is taken to be the center of the beam spot which is $20.6 \mu \mathrm{m}$ away from the transmitarray and the designed focal length is $21 \mu \mathrm{m}$, (b) the complex magnitude of the transmitted E-field across the focal plane. The incident field magnitude is $\sqrt{2} \mathrm{~V} / \mathrm{m}$ and the peak magnitude of the focused field is $3.89 \mathrm{~V} / \mathrm{m}$. The transmitarray has a focusing power of 2.75 times.

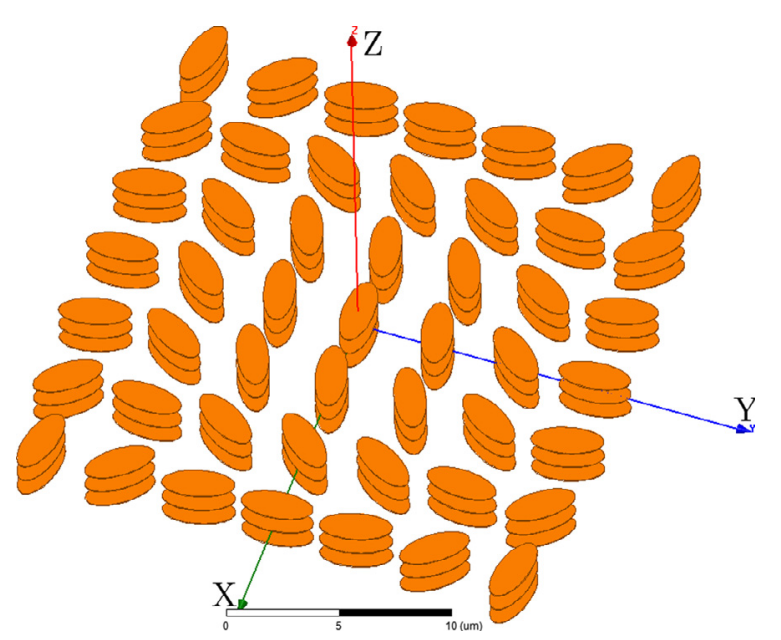

Figure 9. Full-wave simulation model of a 2D-focusing transmitarray lying in the $X$-Y plane.

efficiency of the single unit cell presented in Section 2.2. Therefore, once the efficiency of a single unit cell is maximized, the efficiency of the entire transmitarray is maximized as well. Compared to prior art, the design process for this refraction lens is greatly simplified and is more scalable.

\subsection{Application: 1D-focusing}

A focusing transmitarray can be created by using the unit cells to compensate different free space path delays as shown in Figure 6. By setting a desired focal length, each unit cell is rotated accordingly. The design of the 1D-focusing transmitarray is shown in Figure 7. With a normal incident plane wave, the magnitude of the transmitted field is shown Figure 8a. The focal spot of this transmitarray is designed to be $21 \mu \mathrm{m}$ or $2 \lambda$ away from the transmitarray. The simulated focal spot is taken to be the center of the beam spot that is $20.6 \mu \mathrm{m}$ away.
The simulated result is in a good agreement with our calculation. The complex magnitude of the E-field across the focal plane is plotted in Figure 8b. The incident field magnitude is $\sqrt{2} \mathrm{~V} / \mathrm{m}$. The peak magnitude of the focused field is $3.89 \mathrm{~V} / \mathrm{m}$. The transmitarray has a focusing power of 2.75 times. The half power beam width is $5.2 \mu \mathrm{m}$, or half-wavelength.

\subsection{Application: 2D-focusing}

A small 2D-focusing transmitarray with unit cells arranged in a $7 \times 7$ square array is shown in Figure 9. The full-wave simulation result is shown in Figure 10 where the focusing effect is clearly observable. Unfortunately it is not possible to simulate larger transmitarray designs due to the large computational resources needed. For larger designs, we can apply Love's equivalence principle [15] to do a near-field to far-field transformation. The total E-field and $\mathrm{H}$-field of the single unit cell are sampled on two sampling planes as shown in Figure 11. One sampling plane is above the top elliptical patch and one sampling plane is below the bottom elliptical patch. The sampling planes are parallel to the $X-Y$ plane and reside in the nearfield region of the radiating patches. Equivalent electric and magnetic surface currents $J_{\mathrm{S}}$ and $M_{\mathrm{S}}$ can be computed from those sampled fields. The far-field radiation pattern of this single unit can then be computed from those equivalent surface currents. By stitching together the surface currents from all the unit cells, the field pattern of the entire array can be calculated. Figures $12 \mathrm{a}$ and $12 \mathrm{~b}$ show the complex magnitudes of the E-field in $X-Y$ and $Y-Z$ plane for a square array with $81 \times 81$ unit cells. The focusing beam patterns match well those from the full-wave simulation of the smaller $2 \mathrm{D}$ array.

\subsection{Application: polarization discriminator}

In addition to the refraction and focusing applications presented above, the refraction lens presented in Section 2.3 can 


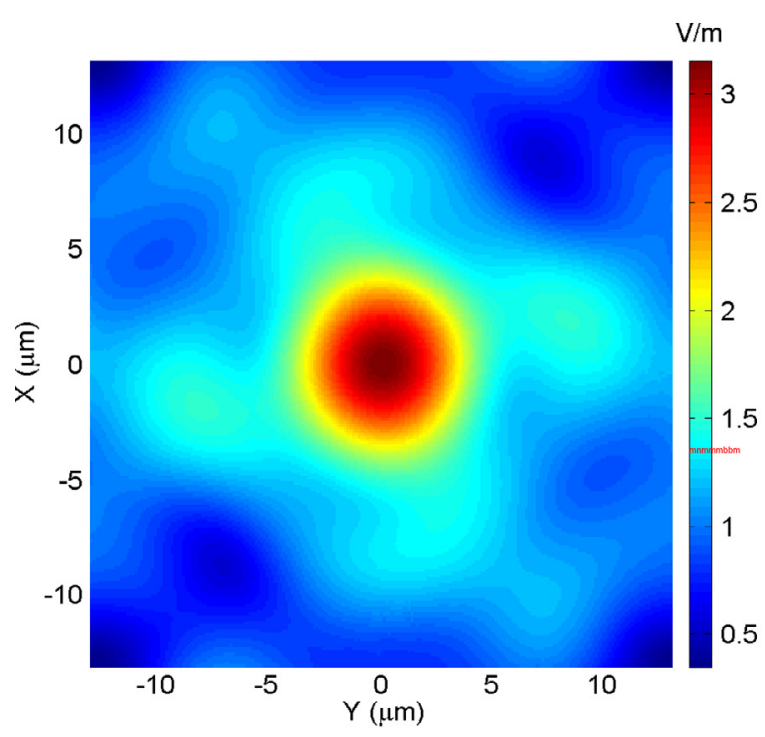

(a)

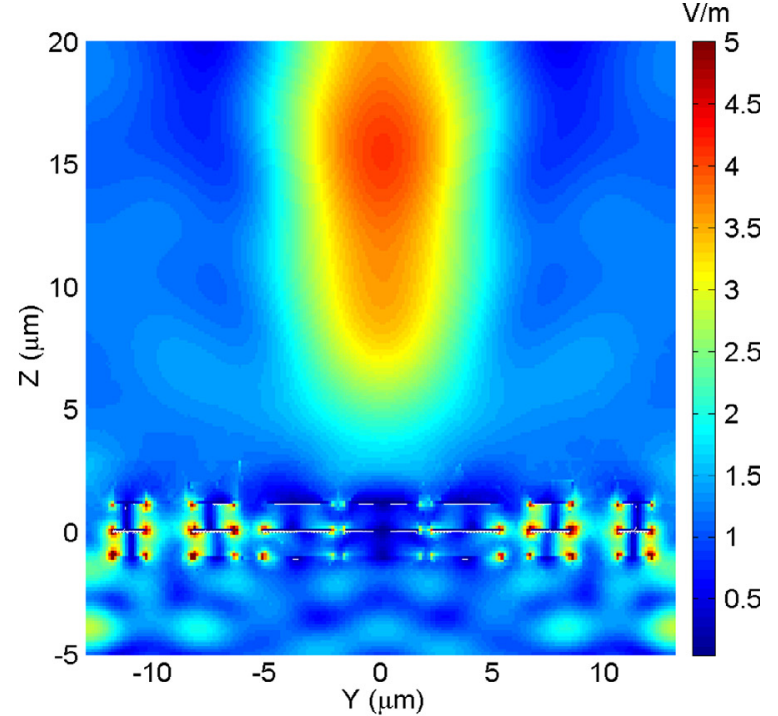

(b)

Figure 10. Incident plane wave has a magnitude of $\sqrt{2} \mathrm{~V} / \mathrm{m}$. (a) Complex magnitude of E-field on the $X$ - $Y$ plane, (b) complex magnitude of E-field on the $Y-Z$ plane.

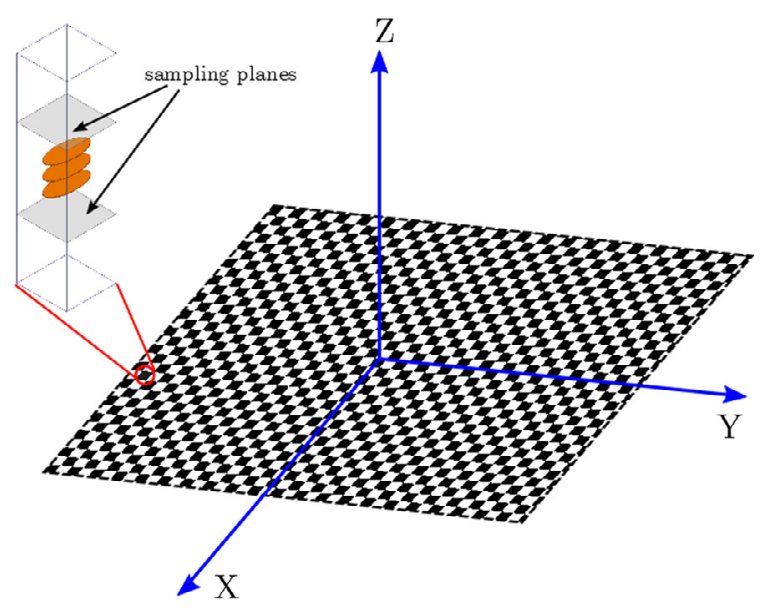

Figure 11. Surface currents $J_{\mathrm{S}}$ and $M_{\mathrm{S}}$ are computed from the sampled E-field and $\mathrm{H}$-field on the sampling planes that reside in the near field of the radiating patches. By stitching together the surface currents from all the unit cells in the array, the far-field pattern can be computed.

be used to discriminate the orientation of a normally incident wave with linear polarization. To begin with, in the theory part of this paper, if the handedness of the incident $\mathrm{CP}$ wave changes, it is not difficult to see that the phase delay due to the rotation of the unit cell reverses its sign with equal magnitude. Hence, if the refraction lens introduces a positive phase gradient for a normally incident RHCP wave, it will introduce a negative phase gradient for a normally incident LHCP wave. As shown in Figure 13, an incident LHCP wave is refracted into a RHCP wave at an angle of $-\theta$, and an incident RHCP wave is refracted into a LHCP wave at an angle of $+\theta$.

A normally incident linearly polarized wave can be decomposed into a summation of a LHCP and a RHCP wave.
For example, an $X$-polarized and a $Y$-polarized wave with unity magnitude can be written as:

$$
\begin{gathered}
\hat{x}=\frac{1}{2}(\hat{x}-j \hat{y})+\frac{1}{2}(\hat{x}+j \hat{y}) \\
\hat{y}=-j\left[\frac{1}{2}(\hat{x}-j \hat{y})-\frac{1}{2}(\hat{x}+j \hat{y})\right]
\end{gathered}
$$

As a result, for $X$-polarized and $Y$-polarized waves, the refracted waves consist of both RHCP and LHCP waves, but there is a $180^{\circ}$ phase difference between the RHCP and LHCP waves depending on whether the incidence is oriented along the $X$ or the $Y$-axis. This phase difference results to different interference patterns of the transmitted waves.

To obtain an analytical expression of the interference pattern due to different orientations of a linearly polarized incident wave, we can assume that the two transmitted waves are two $\mathrm{CP}$ plane waves with propagation vectors $\overrightarrow{k_{L}}=k \sin \theta \hat{x}+k \cos \theta \hat{z}$ and $\overrightarrow{k_{R}}=-k \sin \theta \hat{x}+k \cos \theta \hat{z}$, where $\theta$ is the refraction angle with respective to the $z$-axis as shown in Figure 13. Then the relation between the incident and transmitted CP waves can be mapped as in equations (11) and (12).

$$
\begin{aligned}
& (\hat{x}-j \hat{y}) \rightarrow(\cos \theta \hat{x}+j \hat{y}-\sin \theta \hat{z}) e^{-j(k x \sin \theta+k z \cos \theta)} \\
& (\hat{x}+j \hat{y}) \rightarrow(\cos \theta \hat{x}-j \hat{y}+\sin \theta \hat{z}) e^{j(k x \sin \theta-k z \cos \theta)}
\end{aligned}
$$

With the help of equations (9) and (10), the transmitted waves for normally incident linearly polarized waves with fields polarized along the $\hat{x}$ and $\hat{y}$ directions can be mapped as in equations (13) and (14), assuming that the field magnitudes are unity and dropping the constant phase term $e^{-j k \cos \theta z}$. 


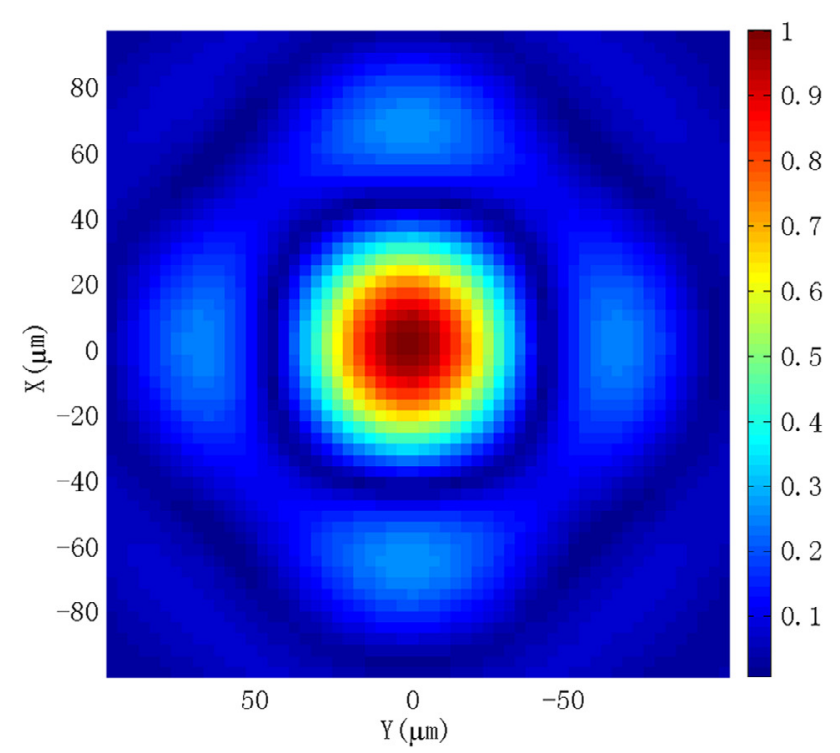

(a)

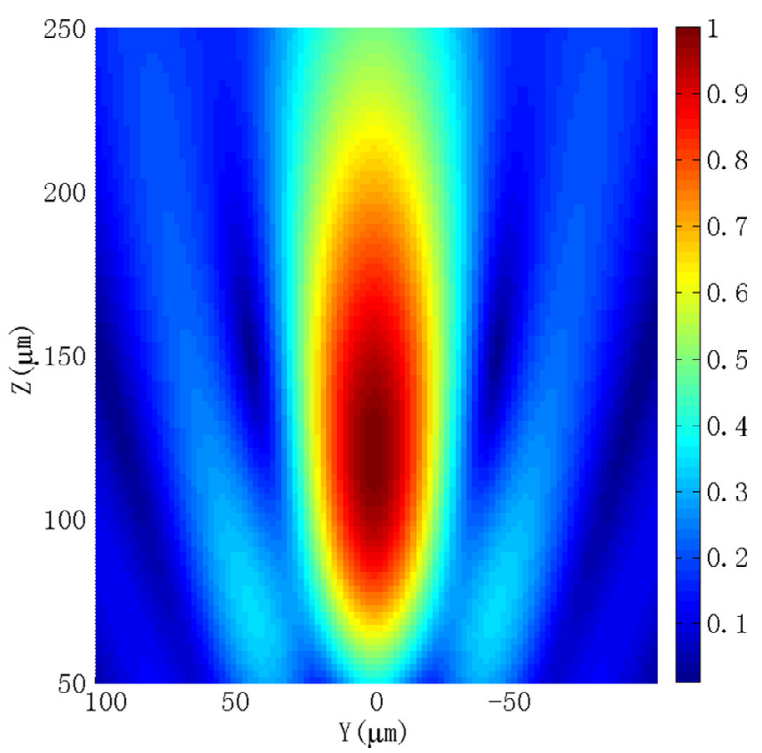

(b)

Figure 12. (a) Normalized complex magnitude of E-field on the $X-Y$ plane, (b) normalized complex magnitude of E-field on the $Y$ - $Z$ plane.

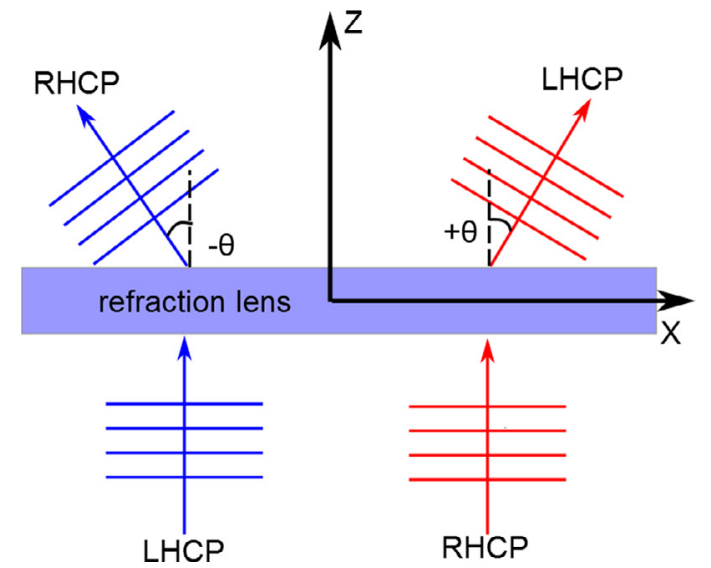

Figure 13. For a $1 \mathrm{D}$ refraction lens with phase gradient in the $x$-direction, the refraction angles for the transmitted plane waves are equal but opposite in sign for LHCP and RHCP incident waves.

$$
\begin{aligned}
\hat{x} \rightarrow & \hat{x} \cos \theta \cos (k x \sin \theta)+\hat{y} \sin (k x \sin \theta) \\
& +j \hat{z} \sin \theta \sin (k x \sin \theta) \\
\hat{y} \rightarrow & \hat{x} \cos \theta \sin (k x \sin \theta)-\hat{y} \cos (k x \sin \theta) \\
& -j \hat{z} \sin \theta \cos (k x \sin \theta)
\end{aligned}
$$

Finally, for a linear polarization with any orientation and unity magnitude, we can decompose it as in equation (15),

$$
\vec{E}_{\text {inc }}=\hat{x} \cos \phi+\hat{y} \sin \phi
$$

where $\phi$ is the angle between the $x$-axis and orientation of the electric field. By substituting equations (13) and (14) into equation (15), the transmitted field $\vec{E}_{t}$ due to $\vec{E}_{\text {inc }}$ can be written as:

$$
\begin{aligned}
\vec{E}_{t}(\phi, x, \theta)= & \hat{x}\{\cos \phi \cos \theta \cos (k x \sin \theta) \\
& +\sin \phi \cos \theta \sin (k x \sin \theta)\} \\
& +\hat{y}\{\cos \phi \sin (k x \sin \theta)-\sin \phi \cos (k x \sin \theta)\} \\
& +j \hat{z}\{\cos \phi \sin \theta \sin (k x \sin \theta) \\
& -\sin \phi \sin \theta \cos (k x \sin \theta)\}
\end{aligned}
$$

The full setup for the proposed polarization discriminator is shown in Figure 14. If a normally incident linearly polarized wave with orientation angle $\phi$ with respect to the $x$-axis passes through a refraction lens with a phase gradient in the $x$-direction, the transmitted intensity is $\left|\vec{E}_{t}\right|^{2}$ with spatial variations in the $x$-direction. The positions of the peaks and troughs of this intensity pattern depend on the orientation angle $\phi$. By simply placing photo-detectors behind the lens to measure the spatial variation of the transmitted intensity, the orientation angle of the normally incident linearly polarized light can be determined.

We can illustrate this concept by using an example. The refraction lens design in Section 2.3 has a designed refraction angle $\theta$ of $-17^{\circ}$. Using this refraction angle, we can plot the intensity $\left|\vec{E}_{t}\right|^{2}$ vs. $x$ for various orientation angles $\phi$ as shown in Figure 15. The transmitted intensities have sinusoidally varying spatial distributions. As the orientation angle $\phi$ of the incident linearly polarized wave changes, the positions of the peaks and troughs of the transmitted intensity distributions change correspondingly.

Fullwave simulated results using the refraction lens presented in Section 2.3 are shown in Figure 16. The intensity distributions are not pure sinusoids as in the theoretical case. This is because the transmitted wave is not a perfect plane wave; 


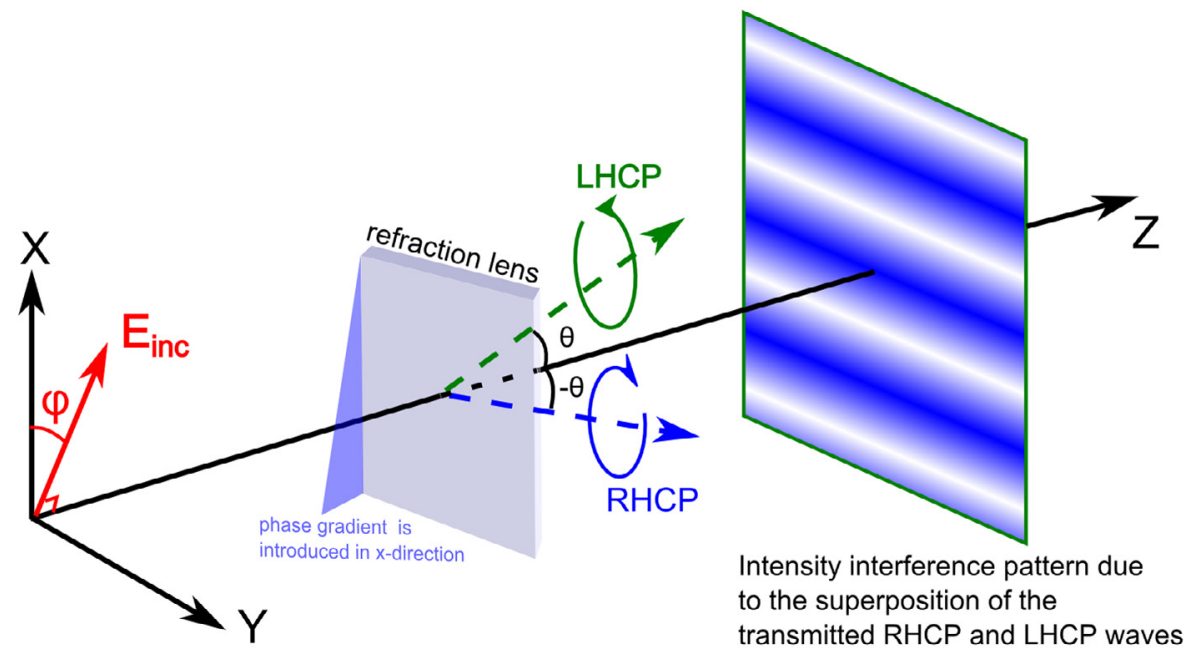

Figure 14. If a linearly polarized wave $E_{\text {inc }}$ with orientation angle $\phi$ with respect to the $x$-axis impinges normally on a refraction lens having a phase gradient in the $x$-direction, the $x$-positions of the peaks and troughs of the transmitted intensity pattern will depend on the orientation angle $\phi$.

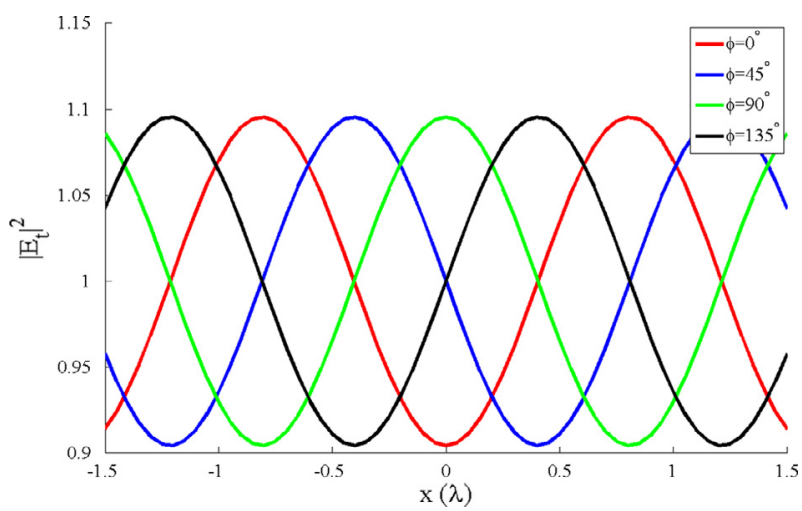

Figure 15. The theoretical result of the transmitted intensity $\left|\vec{E}_{t}\right|^{2}$ vs. the spatial position $x$ for various orientation angles $\phi$ of a linearly polarized incident plane wave. The spatial positions of the peaks and troughs vary correspondingly with the orientation angle.

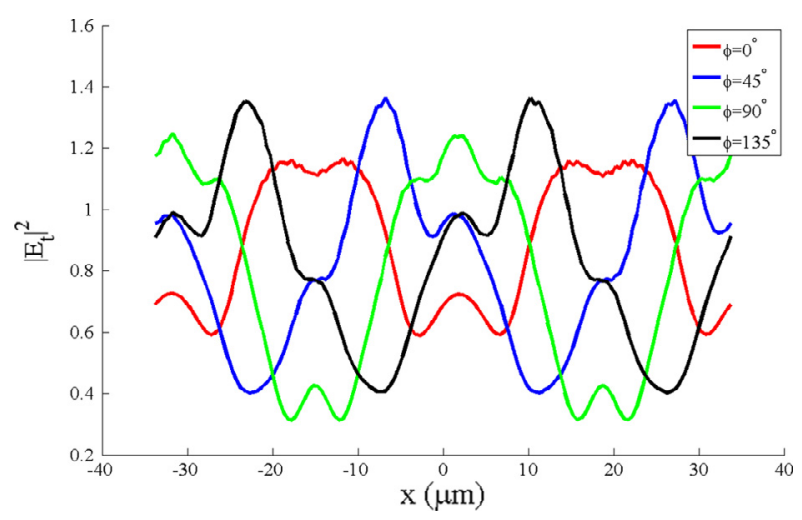

Figure 16. The simulated result of the intensity $\left|\vec{E}_{t}\right|^{2}$ vs. spatial position $x$ for various orientation angles $\phi$. Since the reconstruction of the plane wave is not perfect, the intensity distribution is not sinusoidal as in the theoretical result. However, the relative positions of the peaks and troughs match well with those of the theoretical results.

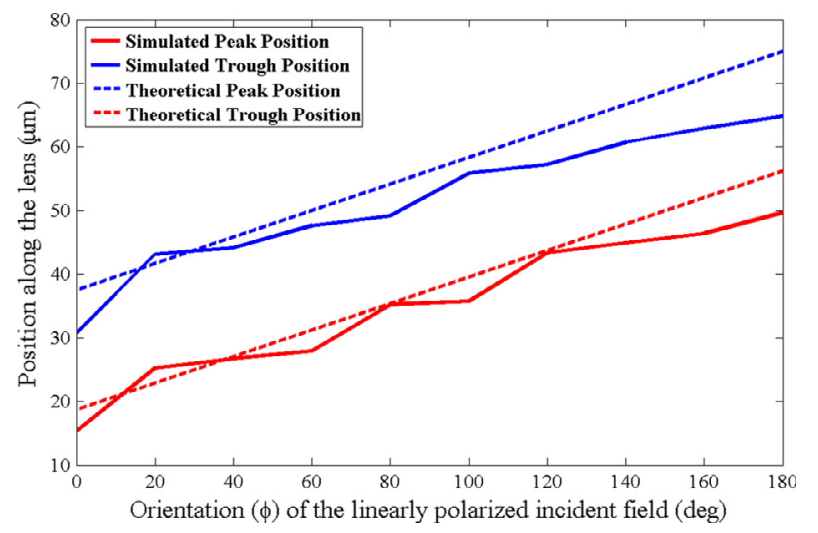

Figure 17. Theoretical and simulated peak and trough positions along the lens with respect to the orientation angle of a linearly polarized incident light.

there are distortions due to imperfect $\mathrm{CP}$ plane-wave reconstruction. There are two major factors that affect the planewave reconstruction. The first is due to the loss of the unit cell. The unit cells consist of three elliptical patches. Upon normal incidence, the current induced along the longer axis of the elliptical patch experiences greater loss compared to the current induced along the shorter axis. Hence, the transmitted fields along the two axes of the elliptical patch have different magnitudes. This can be observed in Figure $2 b$. Due to the difference in transmission magnitudes, the transmitted plane wave is elliptically polarized instead of circularly polarized. Second, the transmission property of each unit cell is characterized under the assumption of infinite periodicity. However, once the unit cells are sequentially rotated, the periodicity is lost. The mutual coupling between patches in a periodic arrangement is different than that in an aperiodic arrangement. In fact, the mutual coupling will vary from cell to cell. Due to this variation in mutual coupling, the reconstructed plane wave experiences a nonlinear amplitude and phase delay along the lens. Hence, the intensity shape in Figure 16 is not exactly 
sinusoidal. However, the simulated relative positions of the peaks and troughs with respect to the various orientations in $\phi$ match well with the theoretical ones. Capturing these relative positions is the most important factor for the functionality of the proposed linear polarization discriminator. The detectors behind the lens only need to determine the relative positions of the highest and lowest received intensity in order to determine the angle of the orientation. The magnitude variation between the peaks and troughs are less important. Figure 17 shows the theoretical and simulated positions for one peak and one trough with respect to the orientation angle $\phi$. The positions are sampled every $20^{\circ}$ change in $\phi$. It can be seen, that the simulated results follow the theoretical results fairly well. This concept can result to a very simple and compact imaging system for polarimetry applications. The conventional methods for polarimetry application $[16,17]$ use a supercell consisting of four wire grid micropolarizers oriented at $0^{\circ}$, $45^{\circ}, 90^{\circ}$ and $135^{\circ}$. By using CCD sensors behind each micropolarizer to measure the transmitted intensity, the Stokes parameters are extracted to estimate the angle of the orientation. However, each micropolarizer suffers different degrees of polarization mismatch which leads to power loss. If we replace the wire grid micropolarizers in the supercell with our refraction lens, also oriented at $0^{\circ}, 45^{\circ}, 90^{\circ}$ and $135^{\circ}$, we gain two important advantages. First, each refraction lens offers some degree of estimation of the orientation angle; hence, with four refraction lenses used in combination, we can have a much better estimation of the Stokes parameter. Second, the refraction lens has no polarization mismatch, almost all the power is transmitted to the detectors and hence the SNR of the system is improved. As a result, we should be able to achieve resolution beyond the conventional method.

\section{Conclusion}

In summary, this paper presents a simple CP optical transmitarray family comprising rotated unit cells. Each unit cell consists of three stacked elliptical metallic patches. The transmission phase of the light is only dependent on the rotation angle of the unit cell in a simple deterministic manner. Thus, by arranging the unit cells with particular orientations, transmitarrays for LWIR light refraction and focusing have been demonstrated. The resulting structures are completely planar and optically thin and offer a transmission efficiency above $70 \%$ (which can be improved by stacking more metallic patches) over the frequency range of $28-32 \mathrm{THz}$, which is equivalent to $14 \%$ bandwidth. Lastly, by using a flat refraction lens made using this approach, the orientation angle of a normally incident linearly polarized light can be detected by simply measuring the transmitted intensity pattern. This constitutes a novel and compact polarization discriminator device.

\section{Implication and influences}

The presented concept of realizing IR transmit-arrays for CP light using stacked rotated metallic patches could have several important implications to the corresponding field and open the door to new applications. First, the introduced phase delay associated with each unit cell is only dependent on the rotation angle of the unit cell instead of its linear geometrical parameters. To refract the incident light to different angles or focal lengths one only has to rearrange the unit cells to a different orientation instead of redesigning each and every unit cell in the array. Thus, this approach offers much more flexibility to the design process. Second, the design is easily realizable with current fabrication technology which is crucial for integration. In addition, the materials used in this design have a high laserinduced damage threshold (LIDT) which is very attractive for high-power laser machining applications. Moreover, the concept of using a so-realized refraction lens to detect the orientation of a linearly polarized incident light introduces a very simple and compact solution for potential infrared polarimetry applications. This polarization discriminator does not suffer from power loss due to polarization mismatch as in conventional designs. Finally, it should be noted that the proposed structures are scalable to other optical spectral ranges as long as the metal used remains reasonably opaque.

\section{References}

1. N. Yu, P. Genevet, M.A. Kats, F. Aieta, J. Tetienne, F. Capasso, Z. Gaburro, Light propagation with phase discontinuities: generalized laws of reflection and refraction, Science 6054 (2011) 333-337.

2. F. Aieta, P. Genevet, M.A. Kats, N. Yu, R. Blanchard, Z. Gaburro, F. Capasso, Aberration-free ultrathin flat lenses and axicons at telecom wavelengths based on plasmonic metasurfaces, Nano Lett. 12 (2012) 4932-4936.

3. X. Ni, S. Ishii, A.V. Kildishev, V.M. Shalaev, Ultra-thin, planar, Babinet-inverted plasmonic metalenses, Light: Science \& Applications e72 (2013) 1-6.

4. F. Monticone, N.M. Estakhri, A. Alù, Full control of nanoscale optical transmission with a composite metascreen, Phys. Rev. Lett. 110 (2013) 203903.

5. C. Pfeiffer, A. Grbic, Cascaded metasurfaces for complete phase and polarization control, Appl. Phys. Lett. 102 (2013) 231116.

6. B. Memarzadeh, H. Mosallaei, Array of planar plasmonic scatterers functioning as light concentrator, Opt. Express 36 (2011) 2569-2571.

7. S. Larouche, Y. Tsai, T. Tyler, M. Jokerst, D.R. Smith, Infrared metamaterial phase holograms, Nature Mater. 11 (2012) $450-454$.

8. X. Chen, L. Huang, H. Muhlenbernd, G. Li, B. Bai, Q. Tan, G. Jin, C. Qiu, S. Zhang, T. Zentgraf, Dualpolarity plasmonic metalens for visible light, Nature Comm. 3 (2012) 1-6.

9. J. Huang, R.J. Pogorzelski, A Ka-band microstrip reflectarray with elements having variable rotation angles, IEEE Trans. Antennas Propag. 46 (1998) 650-656.

10. R.H. Phillion, M. Okoniewski, Lenses for circular polarization using planar arrays of rotated passive elements, IEEE Trans. Antennas Propag. 59 (2011) 1217-1227.

11. Y. He, G.V. Eleftheriades, Design of thin infrared quarter-wave and half-wave plates using antenna-array sheets, Opt. Express 21 (2013) 24468-24474. 
12. V.G. Niziev, A.V. Nesterov, Influence of beam polarization on laser cutting efficiency, J. Phys. D: Appl. Phys. 32 (1999) 1455-1461.

13. J.S. Tyo, D.L. Goldstein, D.B. Chenault, J.A. Shaw, Review of passive imaging polarimetry for remote sensing applications, Appl. Opt. 45 (2006) 5453-5469.

14. R.M. Wood, Laser-induced damage of optical materials, CRC Press, 2003.

15. A.E.H. Love, The integration of the equations of propagation of electric waves, Philos. Trans. R. Soc. Lond. A 197 (1901) 1-45.
16. F. Sadjadi, Polarimetric IR automatic target detection and recognition, 1996 IEEE International Conference on Acoustics, Speech, and Signal Processing, 1996. ICASSP-96. Conference Proceedings, Atlanta, GA, 4 (1996) 2140-2143.

17. G.P. Nordin, J.T. Meier, P.C. Deguzman, M.W. Jones, Micropolarizer array for infrared imaging polarimetry, J. Opt. Soc. Am. A 16 (1999) 1168-1174.

Cite this article as: He Y \& Eleftheriades GV: Rotated infrared antenna transmitarray for the manipulation of circularly polarized wavefronts. EPJ Appl. Metamat. 2014, 1,8. 